\title{
Mirror-Aided Non-LOS VLC Channel Characterizations with A Time-Efficient Simulation Model
}

\author{
Yuhui Wu · Pieter Audenaert - Mario Pickavet · Didier Colle
}

Received: date / Accepted: date

\begin{abstract}
The emerging cost-efficient visible light communication (VLC)-based indoor wireless network requires an economical solution for backhaul transmission. Non-lineof-sight (non-LOS) VLC links are generally applicable candidates to set up a backhaul network without rearrangement of existing lighting fixtures. Here, we describe non-LOS channels aided by the first-order specular reflection from mirrors, which can be used to overcome the multipath effect of purely diffuse non-LOS channels. Characterizations of purely diffuse and mirror-aided non-LOS channels are conducted with a time-efficient simulation model based on an iterative algorithm. Any bounce of reflections combined with specular and diffuse reflections can be simulated using the proposed iterative VLC model in polynomial time. Simulation results show that mirror-aided non-LOS channels outperform purely diffuse non-LOS links regardless of the link configuration. The effect of concentration and directionality of non-LOS VLC channels are also shown and discussed.
\end{abstract}

Keywords visible light communication - backhaul network · channel modeling $\cdot$ diffuse and specular reflection

\section{Introduction}

Visible light communication (VLC) is an emerging technology to address the bandwidth bottleneck of the radio frequency (RF) spectrum. A VLC-based indoor wireless network uses existing light-emitting-diode (LED) lamps for simultaneous illumination and data transmission. The prospect of ubiquitous VLC applications owing to the wide

Yuhui $\mathrm{Wu}$

Ghent University-IMEC, Technologiepark 126, B-9052 Gent (Zwijnaarde)

Tel.: +3293314977

E-mail: yuhui.wu@ugent.be usage of LEDs for indoor lighting as well as the potential of reliable gigabit transmission makes VLC a promising technique for seamless and high-speed indoor wireless data transmission. A VLC-based indoor wireless network uses LED lamps as access points (APs), each of which provides access transmission within a small cell which is commonly referred to as an attocell. Despite the growing scientific interest on access links of VLC attocell networks [1-4], i.e. data transmission from LED modules of APs to user devices, an underlying assumption in those studies is that all APs are wired connected to the Internet. Various wired backhaul approaches have been proposed to be integrated with the VLCbased network, which are shown in Fig. 1(a). Power line communication (PLC) uses the existing electric power grid for the backhaul data transmission $[5,6]$. The main issue is the high interference and the non-white noise [7]. Powerover-Ethernet $(\mathrm{PoE})$ has been proposed for integrating the power supply and the high-speed backhaul transmission in Ethernet cables at the expense of high cost for replacing the existing power wiring [8]. A VLC-based backhaul solution for relay transmission was proposed in [9], where only one gateway employed with six auxiliary LEDs is connected to the Internet via wire. Each auxiliary LED is responsible for the line-of-sight (LOS) link-based backhaul transmission with one auxiliary photodiode (PD) of the AP of an adjacent hexagonal cell. A example of downstream traffic from the gateway to APs is shown in Fig. 1(b). More auxiliary transceivers are required for bidirectional backhaul transmission. Despite the fact that LOS backhaul links are able to support large channel capacity, the arrangement of auxiliary VLC transceivers largely depends on the geometry of cells. Misplacement of auxiliary transceivers could introduce large interference. In addition, heights of APs have to be carefully adjusted in order to maintain the LOS between each pair of auxiliary transceivers. Thus, the design of the LOS link-based wireless backhaul network has a low level 
(a)

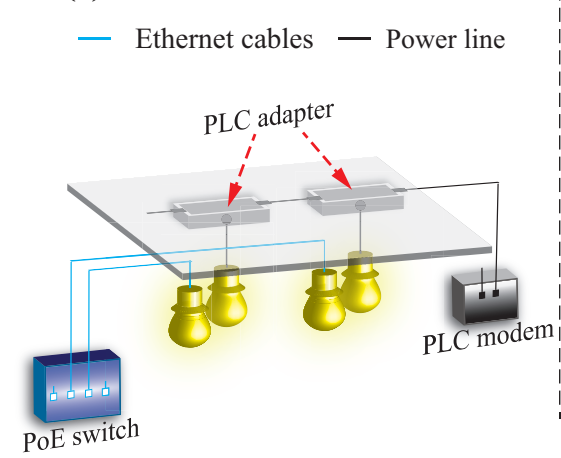

(b)

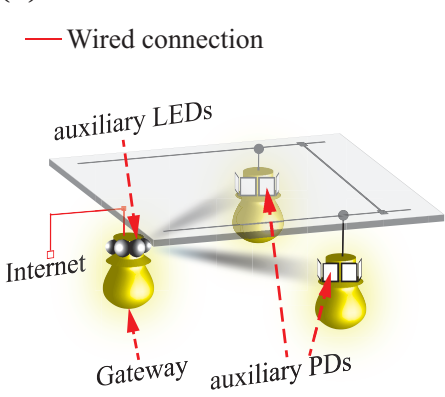

(c)

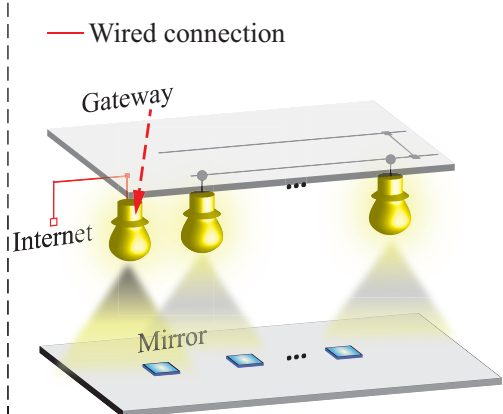

VLC access point

Fig. 1: Backhaul configurations: (a) Wired backhaul links with PoE or PLC; (b) LOS-based wireless backhaul links; (c) Mirror-aided non-LOS wireless backhaul links

of generality. In many realistic environments where a series of ceiling lamps are used for indoor illumination, non-LOS VLC links are more generally applicable. In order to mitigate multipath effects caused by diffuse reflections from interior objects and walls, we propose here a mirror-aided nonLOS VLC link-based backhaul network as shown in Fig. 1 (c). The mirror placed on the floor is used to provide a concentrated and directed first-bounce specularly reflected path from the transmitting AP to the receiving AP such that the channel impulse response is dominated by a specular component. The mirror-aided non-LOS link-based backhaul network necessitates neither replacement of the existing wiring nor strict requirements on the placement of VLC APs. Sizes and positions of mirrors can be easily adjusted according to the application. An added-value of the proposed backhaul solution is that dynamic channel allocation schemes can be easily performed to satisfy different bandwidth requirements on access and backhaul links, as each LED of the VLC AP can be used for simultaneous access and backhaul transmission by allocating different orthogonal frequency division multiplexing (OFDM) subcarriers. Mirror-aided non-LOS links can be foreseen as a low cost and high-performance backhaul solution for the indoor VLC-based wireless network, as the rearrangement of existing lighting fixtures is not required.

Despite fixed positions of lighting fixtures, orientations and concentrations of LED modules and PD modules of each VLC AP can be configured to achieve better channel performance. In order to design a robust backhaul network, extensive research on mirror-aided non-LOS VLC channel characteristics is essential. However, characterization of mirror-aided non-LOS VLC channels cannot be obtained with current simulation models in reasonable time. Analytical methods presented in [10-12] and methods based on Monte-Carlo ray-tracing algorithm [13-15] cannot pro- vide accurate results, as they reduce the time complexity of the simulation at the expense of accuracy. Near-accurate Infrared (IR) channel characterization can be obtained using a well-known recursive computation method $[16,17]$. Based on the recursive method, authors in [18] presented a VLC channel simulation model by adding wavelength-dependent parameters. However, time-complexity is extremely large when increasing the resolution of wavelengths and the accuracy of the results has to be lowered in order to reduce the time-complexity. An iterative method was proposed for IR channel characterization [17,19]. Optimization is needed to apply this method for VLC channel modeling. In addition to the accuracy requirement, the characterization of mirror-aided non-LOS VLC channels should take into account multi-bounce specular reflections. Unfortunately, current iterative methods only model purely diffuse reflections but ignore specular reflections. In order to investigate the performance of mirror-aided non-LOS VLC channels, we present a new iterative model for characterizing wavelengthdependent VLC channels in rooms with Lambertian surfaces and ideal mirrors. Multi-bounce channel impulse responses can be simulated in polynomial time. Simulations are undertaken to investigate the performance of purely diffuse and mirror-aided non-LOS VLC channels as well as the impact of different link configurations on the channel performance. Results show that mirror-aided non-LOS channels outperform purely diffuse non-LOS links regardless of the link configuration. Guidelines for achieving reliable high-speed data transmission using the proposed mirror-aided non-LOS channels are also given. The paper is organized in the following way. The following section introduces theories for analyzing multipath VLC channels. The third section describes the computer implementation of the iterative algorithm. Simulation results and discussions are presented in the fourth section. 


\section{Theoretical approaches}

Intensity-modulation and direct detection (IM/DD) is the most viable technique used in VLC systems, as the main advantage of IM/DD is its simplicity and low-cost [20]. The waveform of an intensity-modulated signal is represented by the instantaneous radiant power of the LED. PDs are commonly used to detect changes in the optical intensity. Light rays radiant from the LED are reflected from walls of the room and surfaces of interior objects. The reflected portion of the optical power depends on the reflectivity of the material at each wavelength. In addition to light rays originated from the transmitter of the concerning VLC channel, a receiver could also receive light radiant from other luminous objects. These luminous objects are considered noise and interfering sources and their contribution to the total received power can be either filtered or mitigated by using multiplexing schemes. In this section, we introduce the method we use to model the simulation environment and multi-bounce reflections. The numerical approach presented in this section is used to iteratively calculate VLC channel impulse responses taking into account different reflecting properties of materials.

2.1 Modeling of the environment

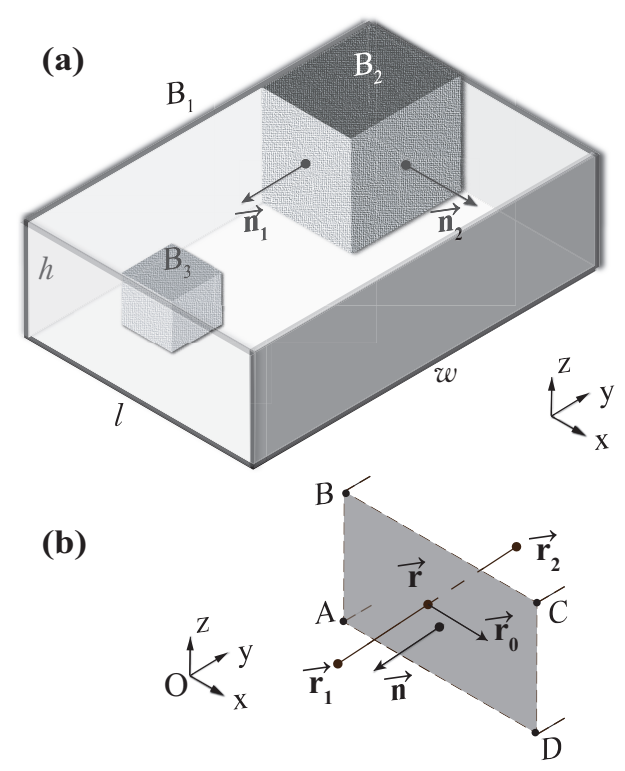

Fig. 2: (a) Geometry of an L-shaped room modeled with a room $\left(B_{1}\right)$, a wall-box $\left(B_{2}\right)$, and an object-box $\left(B_{3}\right)$; (b) A path between two points denoted by $\overrightarrow{\mathbf{r}_{1}}$ and $\overrightarrow{\mathbf{r}_{2}}$ obstructed by a plane at the point $\overrightarrow{\mathbf{r}}$

The simulation environment of indoor VLC channels is a room with optional interior objects (such as bookcases, desks, and people). We encapsulate the room and all objects into axis-aligned bounding boxes. Each bounding box is a rectangular six-sided box having its surfaces oriented in such a way that normal vectors of all surfaces are parallel with axes of the 3-dimensional Cartesian coordinate system. Surfaces oriented towards the positive and the negative direction of the $\mathrm{z}$-axis are defined as the floor and the ceiling of the box, respectively. The other surfaces are defined as sidewalls of the box. The largest box is an empty room with six internal surfaces shown in Fig.2(a) as $B_{1}$. Interior objects $B_{2}$ and $B_{3}$ are modeled as boxes with external surfaces. As the ceiling, the floor, and two sidewalls of $B_{2}$ are overlapping with the floor, the ceiling, and two walls of $B_{1}$, respectively, the simulation environment can be seen as an L-shaped room. All points inside $B_{2}$ are obstructed by the two remaining surfaces (surfaces having orientations $\overrightarrow{\mathbf{n}_{1}}$ and $\overrightarrow{\mathbf{n}_{2}}$ ).

The LOS path of a light ray is modeled as a line segment bounded by two points representing locations of the light source and the receiver. The LOS path between two points can be obstructed by opaque objects between them. Luminous objects, i.e. other lighting fixtures, are also considered opaque objects. Fig.2(b) illustrates the geometry of an obstructed LOS path. The LOS path is obstructed when the line segment intersects at least one surface. The boundary of each surface is defined by vertices (points A, B, C, and D) of the surface. When the surface is a rectangle and all points have positive coordinates, the boundary can be defined by an initial point (point A) and a terminal point (point C), which are vertices located at the minimum and the maximum Euclidean distance from the origin $\mathbf{O}=(0,0,0)$, respectively. The coordinate of a point on a surface is represented by a vector $\overrightarrow{\mathbf{r}}$ and can be calculated by solving the plane equation of the surface:

$$
\overrightarrow{\mathbf{n}} \cdot\left(\overrightarrow{\mathbf{r}}-\overrightarrow{\mathbf{r}_{0}}\right)=0
$$

where $\overrightarrow{\mathbf{n}}$ is the normal vector of the surface and $\overrightarrow{\mathbf{r}_{0}}$ is the coordinate of an arbitrary point on the surface. A LOS path is represented by a line equation:

$$
\overrightarrow{\mathbf{r}}=\overrightarrow{\mathbf{r}_{1}}+\left(\overrightarrow{\mathbf{r}_{2}}-\overrightarrow{\mathbf{r}_{1}}\right) t, \quad 0 \leq t \leq 1
$$

where $\overrightarrow{\mathbf{r}_{1}}$ and $\overrightarrow{\mathbf{r}_{2}}$ are vectors representing locations of the sender and the receiver, respectively, and $t$ is a scaling factor. As the LOS path is a line segment connecting two points, the range of $t$ is limited to $0 \leq t \leq 1$, such that $\overrightarrow{\mathbf{r}}$ is on the line segment between $\overrightarrow{\mathbf{r}_{1}}$ and $\overrightarrow{\mathbf{r}_{2}}$. The coordinate of the point of intersection $\overrightarrow{\mathbf{r}}$ can be solved by (1) and (2). The LOS path is unobstructed by a surface if no intersecting point is found or the point exceeds the surface's boundary. We use a function $V\left(\overrightarrow{\mathbf{r}_{s}}, \overrightarrow{\mathbf{r}_{r}}\right)$ to describe the visibility of the LOS path from a sender $s$ to a receiver $r$, where $\overrightarrow{\mathbf{r}_{s}}$ and $\overrightarrow{\mathbf{r}_{r}}$ are vectors representing coordinates of $s$ and $r$, respectively. When the path does not intersect with any surface in the room $V\left(\overrightarrow{\mathbf{r}_{s}}, \overrightarrow{\mathbf{r}_{r}}\right)=1$, 
and $V\left(\overrightarrow{\mathbf{r}_{s}}, \overrightarrow{\mathbf{r}_{r}}\right)=0$ when the path is obstructed by at least one surface. As the visibility does not change when $s$ and $r$ are swapped, $V\left(\overrightarrow{\mathbf{r}_{s}}, \overrightarrow{\mathbf{r}_{r}}\right)=V\left(\overrightarrow{\mathbf{r}_{r}}, \overrightarrow{\mathbf{r}_{s}}\right)$.

\subsection{VLC channel impulse response}

An IM/DD channel can be modeled as a baseband linear system:

$Y(t)=X(t) * h(t)$

where $*$ denotes convolution, $X(t)$ and $Y(t)$ are waveforms of the transmitted and received signal represented by optical power, respectively, and $h(t)$ is the channel impulse response. As light rays are reflected by surfaces in an indoor environment, the received signal consists of a large number of copies of the transmitted signal carried by light rays reflected by a different number of surfaces. The magnitude of each received copy is the optical power carried by the light ray. The delay is the traveling time of the light ray from the sender to the receiver. When the transmitted signal $X(t)$ is a unit-area Dirac delta function $\delta(t)$, the impulse response of the VLC channel is the superposition of all received copies of the signal. Each copy is a delayed and scaled version of $\delta(t)$. We categorize the copies according to the total number of reflections before reaching the receiver, which is denoted by $k$. The impulse response is

$h(t) \approx \sum_{k=0}^{K} h^{(k)}(t)$,

where $h^{(k)}(t)$ is the superposition of received signals from light rays reflected $k$ times and $K$ is the maximum number of reflections. When $K$ approaching infinity, $h(t)$ approaches the actual impulse response .

In one of the most well-known simulation models for optical wireless channels, the transmitted optical power and the reflectivity of materials are assumed constant across the near monochromatic spectrum of IR [16]. However, visible LEDs generate white light by superposing the radiant power in different color regions. The spectrum of a white LED is not a uniform distribution across all wavelengths, but rather with large peaks occurring in different regions. In addition, the reflectivity of materials and the responsivity of the PD are not constant across the visible range of the electromagnetic spectrum. Thus, the optical power reflected by multiple surfaces and received by the PD is wavelength-dependent. In order to characterize VLC channels taking into account their spectral properties, we model the transmitted signal as a function of wavelength: $X(t, \lambda)=\Phi_{n}(\lambda) \delta(t)$. Here, $\lambda$ is the wavelength in $\mathrm{nm}$, and $\Phi_{n}(\lambda)=\Phi(\lambda) / P_{s}$ is the normalized spectral power distribution (SPD) in $\mathrm{W} \cdot \mathrm{nm}^{-1}$, where $\Phi(\lambda)$ is the SPD of the LED sender and $P_{s}=\int_{390 \mathrm{~nm}}^{700 \mathrm{~m}} \Phi(\lambda) d \lambda$ is

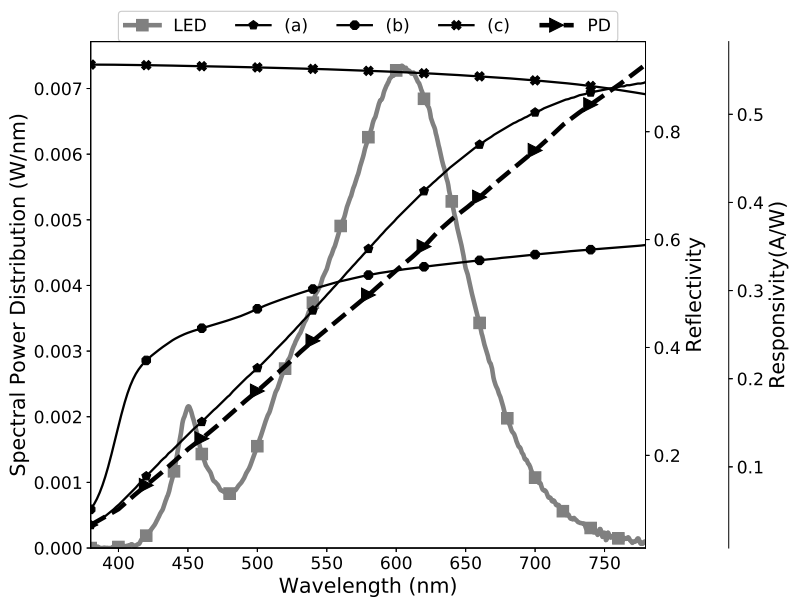

Fig. 3: SPD of LED, responsivity of PD and reflectivity of (a) pine wood at incident angle $10^{\circ}$, (b) white rubberized coating at incident angle $10^{\circ},(\mathrm{c})$ aluminum at normal incidence angle $0^{\circ}$

the total transmitted power of the LED. Fig. 3 shows the normalized SPD of a white Cree BR30 LED lamp, the responsivity of a Thorlabs PD, and the reflectivity of three materials. The spectral characteristic of the LED is obtained from Lamp SPD Database [21]. Over 2400 spectra of natural and artificial materials are available in the ASTER spectral library [22].

For computer simulation, $\delta(t)$ is represented by sending $1 \mathrm{~W}$ optical power in the first time slot. The transmitted power is represented by a 2 -dimensional array $X[t][\lambda]$ :

$X[t][\lambda]=\Phi_{n}[\lambda] \delta[t]$

$X[t][\lambda]$ represents the optical power transmitted at the wavelength sample $\lambda$ during the time slot from $t$ to $t+\Delta t$, where $\Delta t$ is the temporal resolution in ns. The received signal $Y^{(k)}[t][\lambda]$ is the superposition of optical power at the wavelength $\lambda$ and the time slot $t$ of light rays reflected $k$ times. The $k$-bounce impulse response $h^{(k)}(t)$ is calculated by integrating the received signals $Y^{(k)}[t][\lambda]$ over the visible light spectrum.

\subsection{Lambert's cosine law and line-of-sight (LOS) path}

A receiver only detects light rays with incident angles smaller than its field-of-view (FOV). Assuming that the optical power received throughout the active area of the element is constant, the receiver collects the total power of light rays reaching its active area. The received power consists of multiple reflected components and a possibly existing LOS component. The geometry of a LOS path between a point light source $s$ and a receiver $r$ is illustrated in Fig. 4(a). The 
(a)

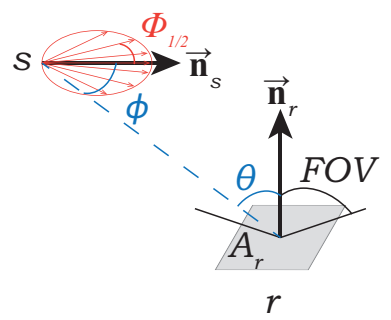

(b)

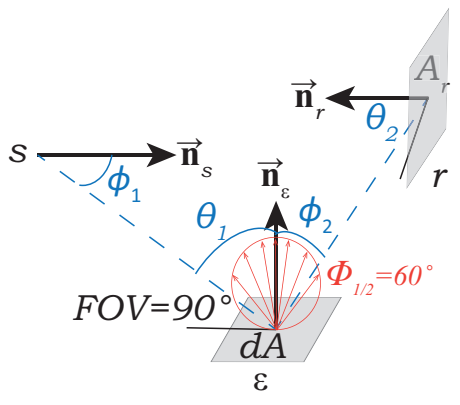

(c)

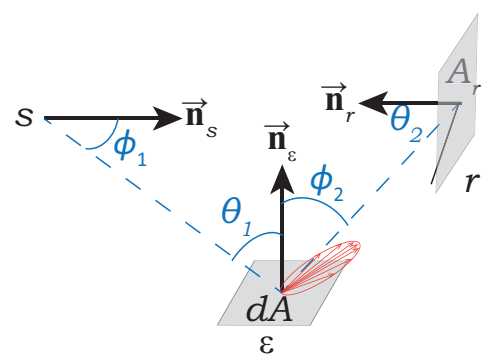

$\varepsilon$

Fig. 4: Geometry of (a) a LOS path from a point light source $s$ to a receiver $r$, (b) a diffusely reflected path from $s$ to $r$ which is reflected off a Lambertian surface with $m=1$ at a point $\varepsilon$, (c) a specularly reflected path from $s$ to $r$ which is reflected off an ideal mirror at a point $\varepsilon$; blue dash lines denote LOS paths while red vectors denote possible directions and lengths of the vectors denote radiant intensity of reflected light rays

optical power received by $r$ is

$P_{r}=\frac{A_{r}}{D^{2}} I_{s} \cos (\theta) \operatorname{rect}\left(\frac{\theta}{F O V}\right)$,

where $\operatorname{rect}(\cdot)$ is a rectangular function:

$\operatorname{rect}(x)= \begin{cases}1, & |x| \leq 1 \\ 0, & |x|>1\end{cases}$

$I_{S}$ is the radiant intensity from the light source $s, F O V$ is the field-of-view of $r, A_{r}$ is the active area of $r$, and $D$ is the Euclidean distance between $s$ and $r$ :

$D=\left\|\overrightarrow{\mathbf{r}_{r}}-\overrightarrow{\mathbf{r}_{s}}\right\|$.

where $\overrightarrow{\mathbf{r}_{s}}, \overrightarrow{\mathbf{r}_{r}}$ represent coordinates of $s$ and $r$, respectively. The incident angle $\theta$ is measured with respect to the orientation of $r$ denoted by the unit-length normal vector $\overrightarrow{\mathbf{n}_{r}}$ of its active area, which can be calculated by

$\cos (\theta)=\frac{\left(\overrightarrow{\mathbf{r}_{s}}-\overrightarrow{\mathbf{r}_{r}}\right) \cdot \overrightarrow{\mathbf{n}_{r}}}{D}$

The radiant intensity $I_{S}$ can be obtained by a generalized Lambert pattern if $s$ is a diffuse radiator (for instance, a LED) obeying Lambert's cosine law [23]. The radiant intensity from a Lambertian surface in the direction with the radiant angle of $\phi$ is

$I_{s}(\phi)=\frac{m+1}{2 \pi} P_{s} \cos ^{m}(\phi) \operatorname{rect}\left(\frac{\phi}{\pi / 2}\right)$,

where $\phi$ is the radiant angle with respect to the orientation of $s$ denoted by a unit-length vector $\overrightarrow{\mathbf{n}_{s}}$ which can be calculated by

$\cos (\phi)=\frac{\left(\overrightarrow{\mathbf{r}_{r}}-\overrightarrow{\mathbf{r}_{s}}\right) \cdot \overrightarrow{\mathbf{n}_{s}}}{D}$

Here, $P_{s}$ is the total radiant power from $s$, the Lambertian order $m=-\frac{\ln (2)}{\ln \left(\cos \left(\Phi_{1 / 2}\right)\right)}$ is an index describing the concentration of $s$, where $\Phi_{1 / 2}$ is the semi angle at half intensity of $s$ and normally given by LED manufacturers, and the rectangular function is used to ensure a non-negative intensity. We use $g_{s, r} d f$ to denote the path loss of a LOS path from a Lambertian sender (i.e. a diffuse radiator) $s$ to a receiver $r$, i.e. the proportion of $P_{r}$ to $P_{S}$ :

$g_{s, r}^{d f}=\frac{(m+1) A_{r}}{2 \pi D^{2}} \cos ^{m}(\phi) \cos (\theta) \operatorname{rect}\left(\frac{\phi}{\pi / 2}\right) \operatorname{rect}\left(\frac{\theta}{F O V}\right)$

The LOS component of the received signal, i.e. the temporal and spectral distribution of the optical power received from the LOS path from $s$ to $r$ is calculated by

$D F(s, r): \quad Y_{s, r}^{d f}[t][\lambda]=g_{s, r}^{d f} V\left(\overrightarrow{\mathbf{r}_{s}}, \overrightarrow{\mathbf{r}_{r}}\right) X_{s}\left[t-\tau_{s, r}\right][\lambda]$,

where $\tau_{s, r}$ is the delay of the LOS path:

$\tau_{s, r}=\frac{D}{c}$,

where $c$ is the speed of light. In order to simplify the notation for further discussions, we use $D F(s, r)$ to denote Equation (13).

\subsection{Modeling of diffuse reflections}

A reflected path can be modeled as having an incident LOS path and a radiant LOS path. The radiant pattern at the reflecting point depends on the material of the reflecting surface. Most rough surfaces are considered Lambertian surfaces and exhibit diffuse reflections. Light rays are reflected in all directions from a reflecting point on a Lambertian surface regardless of the angle of incidence. The radiant intensity can be calculated with (10). Fig. 4(b) illustrates a diffusely reflected path from a light source $s$ to a receiver $r$. The path from $s$ to $\varepsilon$ is called the incident LOS path, and the path from $\varepsilon$ to $r$ is the radiant LOS path. The reflecting point is assumed an ideal Lambertian surface with $m=1$. The optical power is received by an infinitesimal area $d A$ 
around the reflecting point with $F O V=\pi / 2$. The proportion of the radiant power to the received power is defined by the reflectivity of the material.

Multi-bounce received power of a light ray can be obtained by recursively calculating optical power received by each LOS path [16]. However, there are total $N^{k}$ light rays for the $k$-th bounce and thereby in total $N^{k}(k+1)$ calculations of $D F(s, r)$ are required for each $k$-bounce impulse response, where $N$ is the number of reflecting points. The computational effort of the recursive method increases exponentially with $k$. In order to reduce the complexity of calculations without loss of accuracy for a large number of reflections, we calculate the optical power received via diffusely reflected paths iteratively by modeling each reflecting point in the environment as having multiple levels of active senders and receivers. The $k$-th sender and the $k$-th receiver of a reflecting point $\varepsilon$ are denoted by $\varepsilon^{s(k)}$ and $\varepsilon^{r(k)}$, respectively. Similarly, the PD receiver $R$ is modeled as having multiple levels of receivers denoted by $R^{(k)}$. The LED sender $S$ has only one active sender transmitting the signal $X[t][\lambda]$ and is denoted by $S^{(-1)}$. The $k$-th receiver of every reflecting point receives optical power from all $(k-1)$-th senders. The $k$-th sender then radiants the power to all $(k+1)$-th receivers. As the LED and reflecting points are assumed perfect Lambertian surfaces, the radiant power from the $k$-th sender is independent of the direction and the intensity of the light ray from each incident LOS path. Thus, impulse responses with a total number of $K$ bounces can be calculated with $(K+1)$ iterations from 0 to $K$. There is only one sender in the 0 -th iteration. Thus, $(N+1)$ calculations of $D F(s, r)$ are required to calculate the optical power received by the 0 -th receivers of reflecting points and the PD, i.e. $\varepsilon_{i}^{r(0)}, i=0,1, \cdots, N-1$, and $R^{(0)}$. For each additional iteration, $N^{2}$ calculations are required to calculate the power radiant from $N$ senders. The time complexity increases linearly.

\subsection{Modeling of specular reflections}

Glossy surfaces like mirrors exhibit directed and concentrated specular reflections. The radiant intensity from a point on a mirror drops dramatically when the angle between $\phi_{2}$ and $\theta_{1}$ increases as shown in Fig. 4(c), where $\theta_{1}$ is the incident angle of the incident LOS path and $\phi_{2}$ is the radiant angle of the radiant LOS path. It is extremely difficult to iteratively calculate the multipath impulse responses taking into account specular reflections, as the direction and the intensity of the light ray from each radiant LOS path depends on the corresponding incident LOS path. Nonetheless, we can model a specularly reflected path as a LOS path which is "obstructed" by an ideal mirror using the method described in section 2.1. The specular reflection from a perfect mirror is equivalent to a LOS path from the mirror image of $s$ (de- noted by $s^{\prime}$ ) to $r$ which is "obstructed" by the mirror at the intersecting point $\varepsilon$ as illustrated in Fig. 5(a). We assume that all mirrors exhibit perfect specular reflections such that the radiant angle of the radiant path equals the incident angle of the incident path. The path loss of the mirror-obstructed LOS path from $s^{\prime}$ to $r$ can be calculated with $D F\left(s^{\prime}, r\right)$ multiplied with the reflectivity of the mirror. The reflectivity $\rho$ is wavelength-dependent and incident-angle-dependent. The refracted and reflected portion of the light ray is described by the Fresnel equation. Schlick's approximation is a generally used method to approximate the Fresnel equation expressing the reflection of light on a well-oriented facet with less than $1 \%$ error [24], which is formulated as

$\rho(\theta, \lambda) \approx \rho_{0}(\lambda)+\left(1-\rho_{0}(\lambda)\right)(1-\cos (\theta))^{5}$,

where $\theta$ is the incident angle and $\rho_{0}(\lambda)$ is the reflectivity of the material at wavelength $\lambda$ at normal incidence. We use a function $V^{m}\left(\overrightarrow{\mathbf{r}_{s^{\prime}}}, \overrightarrow{\mathbf{r}_{r}}\right)$ to denote the visibility of the mirror-obstructed LOS path. In contrast to the definition of $V\left(\overrightarrow{\mathbf{r}_{s}}, \overrightarrow{\mathbf{r}_{r}}\right)$ in section $2.1, V^{m}\left(\overrightarrow{\mathbf{r}_{s^{\prime}}}, \overrightarrow{\mathbf{r}_{r}}\right)=1$ when the path is obstructed by a mirror, and $V^{m}\left(\overrightarrow{\mathbf{r}_{s^{\prime}}}, \overrightarrow{\mathbf{r}_{r}}\right)=0$ when $r$ cannot "see" the mirror image $s^{\prime}$.

The temporal and spectral distribution of the power received by a mirror-obstructed LOS path is calculated by

$S P(s, r): Y_{s, r}^{s p}[t][\lambda]=g_{s, r}^{s p}[\lambda] V\left(\overrightarrow{\mathbf{r}_{s}}, \overrightarrow{\mathbf{r}_{\varepsilon_{m}}}\right) V\left(\overrightarrow{\mathbf{r}_{r}}, \overrightarrow{\mathbf{r}_{\varepsilon_{m}}}\right) X_{s}\left[t-\tau_{s^{\prime}, r}\right][\lambda]$

where $\varepsilon_{m}$ denotes the reflecting point on the mirror and $g_{s, r}^{s p}[\lambda]=g_{s^{\prime}, r}^{d f} \rho_{s, r}[\lambda] V^{m}\left(\overrightarrow{\mathbf{r}_{s^{\prime}}}, \overrightarrow{\mathbf{r}_{r}}\right)$ is the path loss of the mirrorobstructed LOS path. Similarly, we use $\operatorname{SP}(s, r)$ to denote Equation (16).

The reflecting point on the mirror is considered passive. Thus, each $k$-th receiver detects specularly reflected light rays from $(k-2)$-th senders when there is only one mirror. Multipath impulse responses of mirror-aided non-LOS channels can be iteratively calculated. When multiple mirrors having different orientations are placed as shown in Fig. 5(b), there will be multiple mirror-obstructed LOS paths and thereby multiple reflecting points. The total received power can be calculated by superposing $Y_{s, r}^{s p}[t][\lambda]$ obtained from all mirror images. In a specific case where two mirrors are oriented towards opposite directions, infinite numbers of images will be generated. The time complexity increases with the number of mirror images. In order to simplify the simulation, we assume that all mirrors are placed at the same height with the same orientation, such that each diffusely reflecting point as well as the LED sender $S$ only has one mirror image. A complete study of the combinations of diffuse reflections and specular highlights on glossy surfaces is beyond the scope of this study, but it will be considered in the future work. 
(a)

(b)

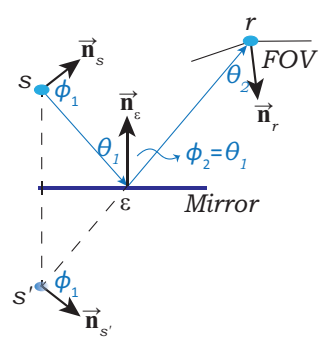

Fig. 5: (a) Geometry of a specularly reflected path (mirror-obstructed LOS path) from $s$ to $r$; $s^{\prime}$ denotes the mirror image of $s, \varepsilon$ denotes the reflecting point; (b) Multi-bounce specular reflections caused by multiple mirror images of $s$

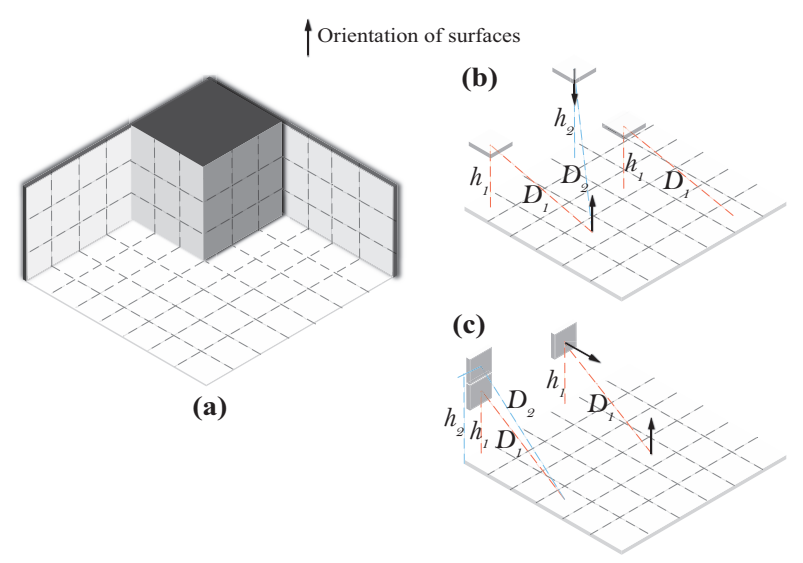

Fig. 6: (a) Differential elements aligned on a Cartesian grid; (b) Elements on two surfaces having opposite orientations; (c) Elements on two intersecting surfaces having perpendicular orientations

\section{Implementation}

Based on the theoretical approaches described in the previous section, we implement a simulation model to iteratively calculate the impulse response of the VLC channel. A major advantage of the iterative method is that the overhead for calculating impulse responses of VLC channels originating from the same sender in the same environment to different receivers is significantly reduced. The iterative algorithm consists of an environment initialization phase, an iterative computation phase, and the final phase for calculating the impulse response.

\subsection{Environment initialization}

As we have discussed in section 2.4 and 2.5, the $k$-bounce impulse response can be calculated by superposing optical power received from all reflecting points. Instead of taking an infinite number of points into account, we divide diffuse surfaces into a grid of differential elements. Each differen-

tial element has an active area of $d A=\Delta d^{2}$, where $\Delta d$ is the spatial resolution. The location (i.e. coordinate) of each differential element as well as locations active senders and receivers of the element are assumed to be the central point of the element. Although the iterative calculation of $k$-bounce impulse responses requires a large number of calculations of $D F(s, r)$ and $S P(s, r)$, it can be seen from (12) and (14) that values of $g_{s, r}^{d f}$ and $\tau_{s, r}$ are independent of the number of reflections. In addition, visibility functions $V\left(\overrightarrow{\mathbf{r}_{s}}, \overrightarrow{\mathbf{r}_{r}}\right)$ and $V^{m}\left(\overrightarrow{\mathbf{r}_{s^{\prime}}}, \overrightarrow{\mathbf{r}_{r}}\right)$ as well as the incident angle-dependent reflectivity only depend on the properties of the environment, i.e. the layout of boxes and reflectivity of surfaces. Thus, we construct lookup tables for $g_{s, r}^{d f}, \tau_{s, r}, V\left(\overrightarrow{\mathbf{r}_{s}}, \overrightarrow{\mathbf{r}_{r}}\right)$, and $\rho_{s, r}[\lambda]$ of all LOS paths between two differential elements in the environment initialization phase. When there are mirrors in the environment, additional lookup tables for $g_{s^{\prime}, r}^{s p}$ and $\tau_{s^{\prime}, r}$ will be constructed. The size of each lookup table is $N_{\varepsilon}^{2}$, where $N_{\varepsilon}$ is the total number of differential elements.

Constructing the lookup table for $g_{s, r}^{d f}$ requires $N_{\varepsilon}^{2}$ calculations of (12). Each calculation consists of several multiplications and summations. We can further reduce the number of calculations for a specific case where all differential elements are aligned on a Cartesian grid where each cell is a unit square having the area of $d A=\Delta d^{2}$ as shown in Fig. 6(a). Since the radiant intensity from a light source is non-negative, $g_{s, r} f f$ is only meaningful when the radiant angle $\phi \leq \pi / 2$. We can categorize Lambertian surfaces with meaningful $g_{s, r}^{d f}$ according to the orientation of one surface relative to the orientation of the other:

- two surfaces having opposite orientations as shown in Fig. 6(b);

- two surfaces having perpendicular (i.e. orthogonal) orientations as shown in Fig. 6(c).

As all differential elements have the same values of $m, d A$, and $F O V, g_{s, r}^{d f}$ is a function of $\overrightarrow{\mathbf{r}_{s}}, \overrightarrow{\mathbf{r}_{r}}, \overrightarrow{\mathbf{n}_{s}}$, and $\overrightarrow{\mathbf{n}_{r}}$. When two surfaces have opposite orientations and vertical distance of $h$ as shown in Fig. 6(b), $g_{s, r}^{d f}$ becomes a function of the relative distance $D$ between $r$ and the projection of $s$. For each value of $h$, there are at maximum $\left(N_{\max }\right)^{2}$ unique values of $D$, where $N_{\max }=L_{\max } / \Delta d$, with $L_{\max }$ is the maximum sidelength of the largest box. For a pair of surfaces having perpendicular orientations, there are $N_{\max }$ unique values of $h$ as shown in Fig. 6(c), thereby $N_{m}^{3}$ unique values of $g_{s, r} d f$. The total number of calculations is reduced to $N_{o p} N_{\max }^{2}+N_{\text {max }}^{3}$. This optimized method is appropriate for simulations when $N_{\varepsilon}$ is very large.

\subsection{Iterative calculations and impulse responses}

In order to obtain the $k$ th bounce impulse response, the signal received by $R^{(k)}$ is calculated. The signal $Y_{R^{(k)}}[t][\lambda]$ is 
the temporal and spectral distribution of the optical power received from all $(k-1)$ th senders. We use $\mathcal{S}^{(k)}$ and $\mathcal{R}^{(k)}$ to denote sets of $k$ th senders and $k$ th receivers, respectively. The iterative algorithm is illustrated in 1 . The size of the array $X_{S}[t][\lambda]$ is $(T \times W)$, where $W=(700 \mathrm{~nm}-390 \mathrm{~nm}) / \Delta \lambda$ is the number of wavelengths, $T=K t_{\max } / \Delta t$ is the number of time slots, (where the temporal resolution $\Delta t=$ $\Delta d /(c \sqrt{2})$ is chosen to be the minimum delay, which is the traveling time of a light ray between central points of two adjacent elements having perpendicular orientations) and $t_{\text {max }}=\left(\sqrt{L_{x}^{2}+L_{y}^{2}+L_{z}^{2}}\right) / c$ is the maximum travelling time of a light ray along the diagonal of the largest box (where $L_{x}, L_{y}$, and $L_{z}$ are side-lengths of the largest box along $x, y$, and $z$ axes, respectively). The $k$-bounce impulse response is calculated with (4), where $Y_{R}^{(k)}[t]=\sum_{\lambda=390 \mathrm{~nm}}^{700 \mathrm{~nm}} Y_{R}^{(k)}[t][\lambda] \Delta \lambda$.

It can be seen from Algorithm 1 that calculations of transmitted signals from active senders of differential elements are independent of $R$. Impulse responses of channels between $S$ and another receiver (i.e. a PD in a different location with different orientation) can be simulated in the same manner by changing the parameters of $R$ in step (6) and (24). Intermediate results $X_{s(k)}[t][\lambda]$ are stored on the hard disk in order to reduce the overhead for calculating the impulse response of multiple channels. We programmed the simulation model in Python 3.5 using open-source packages for calculations and plotting figures. All tables are stored in the form of HDF5 data files on the hard disk. The simulation time and the size of disk files scale with the total number of elements and the maximum number of reflections. We ran simulations on 24-core 64-bit PCs with 24 GB RAM. The simulation of an empty room with a size of $5 \mathrm{~m} \times 5 \mathrm{~m} \times 3 \mathrm{~m}$, $\Delta d=0.2 \mathrm{~m}, \Delta \lambda=10 \mathrm{~nm}$ and $K=7$ took $\sim 6 \mathrm{~min}$ for constructing lookup tables for LOS paths, $\sim 1$ min for the first iteration and $\sim 90 \mathrm{~min}$ for each additional iteration. When the simulation environment and the sender are fixed, the value of $X_{s(k)}[t][\lambda]$ is not changed and can be used to calculate the power received by different receivers. Improving the spatial resolution will lead to large memory consumption and eventually a large amount of simulation time. Authors in [17] found that spatial resolutions of more than five divisions per meter, i.e. with $\Delta d$ smaller than $0.2 \mathrm{~m}$, do not significantly improve the accuracy.

\section{Simulation results}

Channel characterizations of purely diffuse and mirror-aided non-LOS channels are discussed in this section. We model three environments as shown in Table 1. The location of each box denoted by $\overrightarrow{\mathbf{r}}$ is the coordinate of the point located at the minimum distance from the origi and $l, w$ and $h$ denote the side-length of a box along the x-, y-, z-axis, respectively. Environment 1 is designed for simulating purely
Table 1: Simulation environments

\begin{tabular}{c|ccc}
\hline & Environment 1 & Environment 2 & Environment 3 \\
\hline Box & $\overrightarrow{\mathbf{r}}=(0,0,0)$ & $\overrightarrow{\mathbf{r}}=(0,0,0)$ & $\overrightarrow{\mathbf{r}}=(0,0,0)$ \\
(Room) & $l=5 \mathrm{~m}$ & $l=5 \mathrm{~m}$ & $l=5 \mathrm{~m}$ \\
& $w=5 \mathrm{~m}$ & $w=5 \mathrm{~m}$ & $w=5 \mathrm{~m}$ \\
& $h=3 \mathrm{~m}$ & $h=3 \mathrm{~m}$ & $h=3 \mathrm{~m}$ \\
\hline \multirow{2}{*}{ Mirror } & & $\overrightarrow{\mathbf{r}}=(3.2,2.4,0)$ & $\overrightarrow{\mathbf{r}}=(0,0,0)$ \\
& & $l=0.2 \mathrm{~m}$ & $l=5 \mathrm{~m}$ \\
& & $w=0.2 \mathrm{~m}$ & $w=5 \mathrm{~m}$ \\
& & $h=0 \mathrm{~m}$ & $h=0 \mathrm{~m}$ \\
\hline
\end{tabular}

diffuse non-LOS VLC links, while Environment 2 and 3 are used to simulate mirror-aided non-LOS channels. The placement of the mirror depends on locations and orientations of the intended sender and receiver of the mirror-aided non-LOS link in order to provide the first-order specular reflection from the LED sender to the receiver. Ideally, one specularly reflecting point is sufficient to establish a mirroraided non-LOS link. However, the size of a mirror is not infinitesimal and the receiver detects higher-order specular reflections which consist of light rays from differential elements to the receiver reflected by the mirror. Thus, we use a small mirror having the same size as one differential element to simulate an ideal case when the receiver only detects the specular reflection from the sender in Environment 2. The impact of the size of the mirror on channel performance is investigated with an extreme case as Environment 3 where the whole floor is covered by a large mirror such that there aren't any diffuse reflections from the floor and all remaining reflecting points (differential elements) are mirrored.

The materials of surfaces of each box in Table 1 are presented in Table 2. The wavelength-dependent reflectivity of each material has been shown in Fig. 3. In Environment 2 and 3, the floor of the room is covered with a surface which mimics an ideal mirror. Mirrors are assumed to have very thin glass coated with aluminum such that it only exhibits perfect specular reflections on the aluminum coating. In addition to the wavelength-dependent environments, we also simulate environments with constant reflectivity using the same method as in [18], where the reflectivity is a weighted average value across the visible light spectrum:

$\bar{\rho}(\lambda)=\frac{\int_{390 \mathrm{~nm}}^{700 \mathrm{~m}} \Phi_{n}(\lambda) \rho(\lambda) d \lambda}{\int_{390 \mathrm{~nm} m}^{700 m} \Phi_{n}(\lambda) d \lambda}=\int_{390 \mathrm{~nm}}^{700 \mathrm{~nm}} \Phi_{n}(\lambda) \rho(\lambda) d \lambda$

This averaging ensures that the first-order reflected power from each surface is the same as that in the wavelengthdependent environments.

As we have discussed in previous sections, impulse responses of indoor VLC links depend on positions and orientations of the sender and the receiver, as well as intervening reflectors (the environment). The effect of positions of 


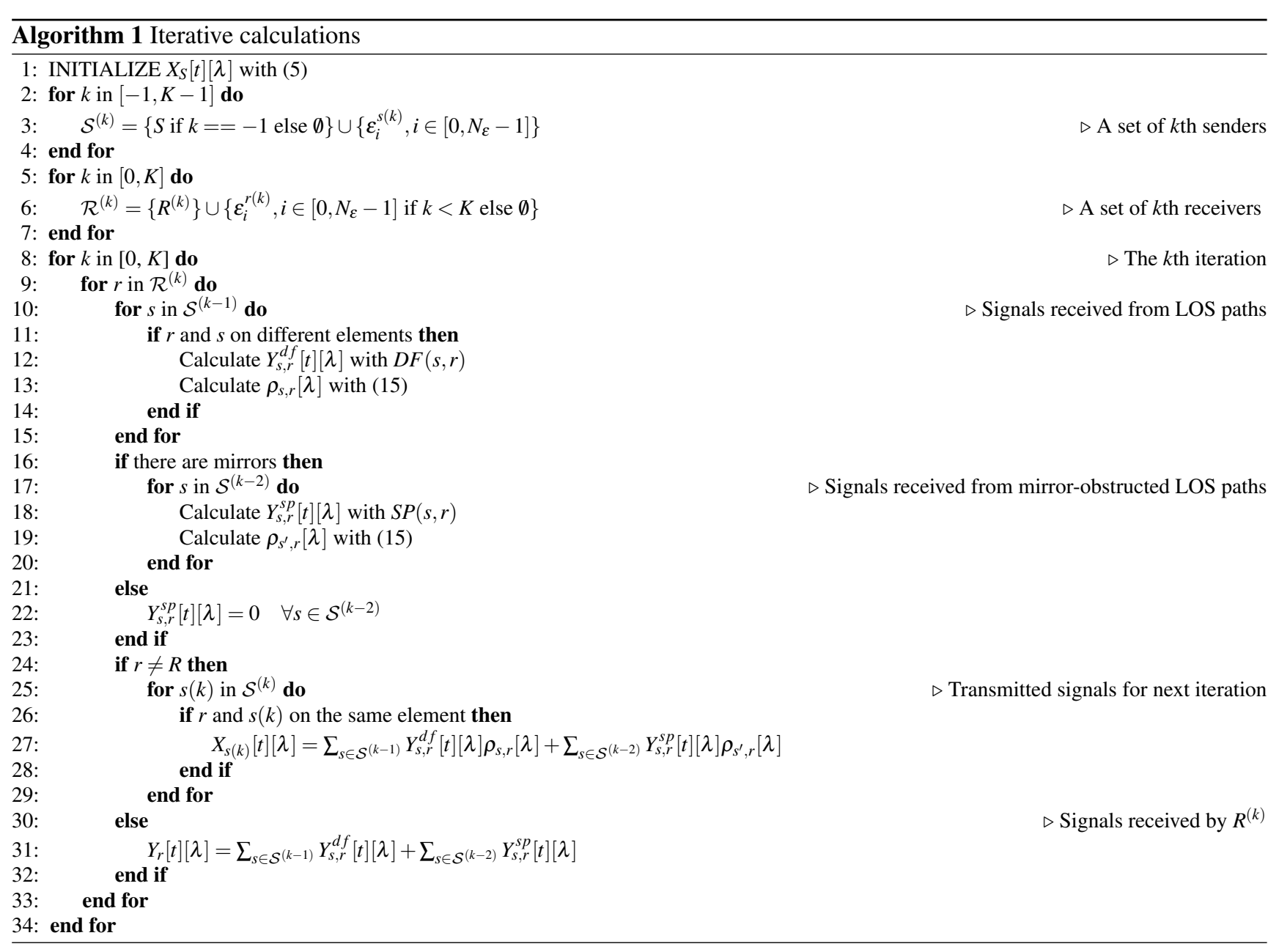

Table 2: Materials and reflectivity of surfaces

\begin{tabular}{l|c|c|c|c}
\hline & \multicolumn{2}{c}{ Room } & \multicolumn{2}{c}{ Mirror } \\
\hline & wavelength dependent & constant & wavelength dependent & constant \\
\hline ceiling & white rubberized coating & 0.52 & - & - \\
floor & pine wood & 0.58 & aluminum coating & 0.91 \\
walls & white rubberized coating & 0.52 & - & - \\
\hline
\end{tabular}

Table 3: Link configurations

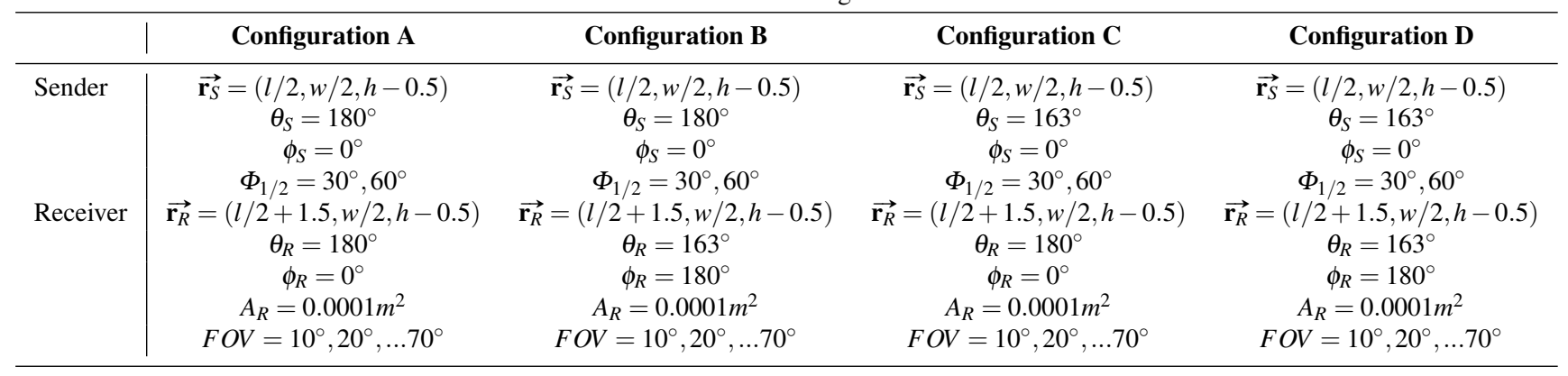


the sender and the receiver on channel impulse responses have been discussed in literature [25,26]. As LEDs of the transmitting AP can be configured to have different orientations and beam-widths and the receiving AP can apply angle-diversity PDs with different viewing angles, it is also worth investigating the effect of configurations on non-LOS VLC links. Therefore, we simulate four non-LOS VLC link configurations as shown in Table 3. Orientations of senders and receivers are represented by unit-length vectors defined by zenith angles $\theta_{S}$ and $\theta_{R}$, azimuth angles $\phi_{S}$ and $\phi_{R}$. The zenith angle ranging from $0^{\circ}$ to $180^{\circ}$ is the angle of a vector measured from the $+z$ axis. A vector on the XOY plane has the zenith angle of $90^{\circ}$. The azimuth angle is a signed angle of the projection of a vector onto the XOY plane with respect to the $+x$ axis. The sign of the angle is so defined that the $+y$ axis has the azimuth angle of $-90^{\circ}$. Four link configurations can be categorized according to orientations of senders and receivers. Senders in configuration C and D are oriented towards a point $\varepsilon_{c}$ on the floor, where $\varepsilon_{c}$ is the first-order specularly reflecting point of mirror-aided nonLOS links. Receivers in configuration B and D are oriented towards $\varepsilon_{c}$. Senders and receivers in other configurations are oriented directly downwards.

\subsection{Multipath effect on non-LOS VLC links}

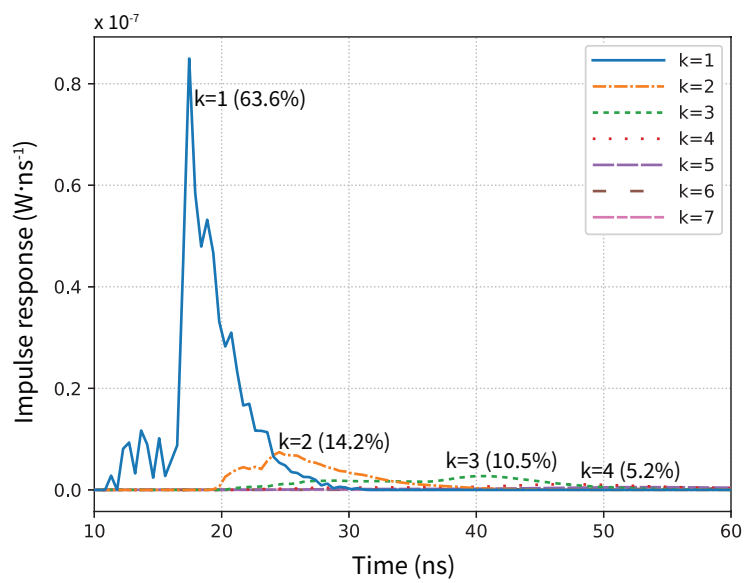

Fig. 7: Multipath impulse responses of one link with configuration A (both senders and receivers are oriented directly downwards) in Environment 1 (purely diffuse); $\Phi_{1 / 2}=60^{\circ}, F O V=70^{\circ}, \Delta d=0.2 \mathrm{~m}$, $\Delta \lambda=20 \mathrm{~nm}, K=7$

Impulse responses of non-LOS channels in three environments as described in table 1 are shown in 7,8 and 9. The location of the small mirror and the orientation as well as the $F O V$ of the receiver are configured such that the receiver is able to detect the first-order specular reflection in Environ-

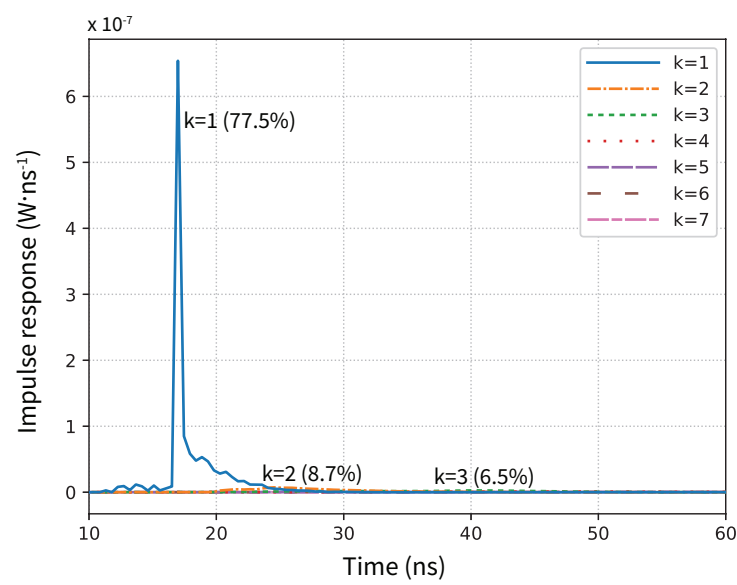

Fig. 8: Multipath impulse responses of one link with configuration A (both senders and receivers are oriented directly downwards) in Environment 2 (mirror-aided, small); $\Phi_{1 / 2}=60^{\circ}, F O V=70^{\circ}, \Delta d=0.2 \mathrm{~m}$, $\Delta \lambda=20 \mathrm{~nm}, K=7$

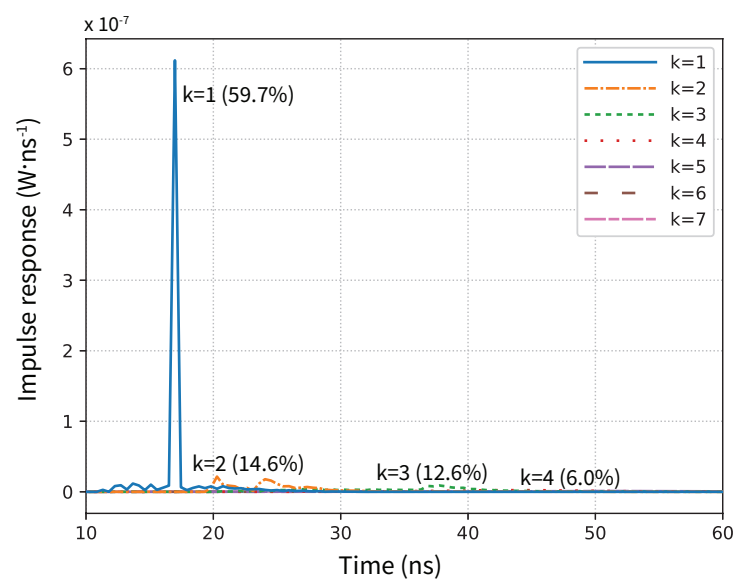

Fig. 9: Multipath impulse responses of one link with configuration A (both senders and receivers are oriented directly downwards) in Environment 3 (mirror-aided, large); $\Phi_{1 / 2}=60^{\circ}, F O V=70^{\circ}, \Delta d=0.2 \mathrm{~m}$, $\Delta \lambda=20 \mathrm{~nm}, K=7$,

ment 2. Impulse responses $h(t)$ are normalized with a factor $10^{-9} / \Delta t$ which correspond to the optical power received per unit time. The total optical power detected by the receiver of the purely diffuse non-LOS link is $988.6 \mathrm{nW}$. The firstorder component carries $63.6 \%$ of the total optical power. The second-order and the third-order components are still significant and broaden the impulse response as shown in Fig. 7. The receiver of the mirror-aided non-LOS link with a small mirror receives in total $1.6 \mu \mathrm{W}$ optical power. It can be seen in Fig. 8 that the first-order component dominates the impulse response. Received power after $40 \mathrm{~ns}$ is no longer significant as the third order and higher-order components contain relatively low power compared to low-order components. When the size of the mirror increases, the total re- 
ceived power is reduced to $1.3 \mu \mathrm{W}$. The second-order and higher-order components become significant as shown in Fig. 9. It is clearly shown that impulse responses of mirroraided non-LOS links have higher peak values and narrower pulses than the impulse response of the purely diffuse nonLOS link. It can be seen that increasing the size of the mirror reduces the peak value of the first bounce $(k=1)$ and increases higher-order received power. The difference in the first-bounce received power is due to the lack of first-order diffuse reflections from the floor which is covered by a mirror in Environment 3. As the specular reflection is directional, the receiver in Environment 3 can only detect one first-order specular reflection from the large mirror on the floor, while one first-order specular reflection and multiple first-order diffuse reflections can be detected by the receiver in Environment 2. Thus, further increasing mirror size cannot improve the total received power when the mirror size is large enough to provide the desired first-order specular reflection. This also explains why the first-order impulse response in Fig. 9 is narrower than that that in Fig. 8. When the number of reflections increases, the receiver in Environment 3 receives more power from higher-order specular reflections. Small peaks in the second-order and the third-order components are likely due to the fact that highly reflective walls reflect a large amount of power.

\subsection{Wavelength dependency of VLC links}

In order to highlight the wavelength dependency of VLC links, we compare VLC links simulated with the proposed iterative model and the model presented in [18] taking into account the responsivity of PD. Similar to the averaged reflectivity obtained with (17), the wavelength-independent responsivity is the weighted average value of the responsivity shown in Fig. 3, which is $0.296 \mathrm{~A} \cdot \mathrm{W}^{-1}$ calculated with (17). Instead of directly comparing simulated impulse responses, two channel parameters - the DC gain and the root-mean-square (RMS) delay spread - derived from channel impulse responses of two models are compared. The DC gain $H 0$ is the channel magnitude response $|H[f]|$ at zero frequency, i.e. $|H[0]|$. In order to highlight the impact of the total number of reflections $K$ on the simulated results, we calculate the channel DC gain $H 0^{(K)}$ when 0 to $K$ bounces are taken into account:

$H 0^{(K)}=\left|\sum_{t=0}^{N_{t}-1} \sum_{k=0}^{K} h_{P D}^{(k)}[t] \Delta t\right|=\left|\sum_{t=0}^{N_{t}-1} \sum_{k=0}^{K} Y_{R}^{(k)}[t][\lambda] R_{P D}[\lambda]\right|$,

where $N_{t}$ is the number of sampled values of the discrete impulse response, $Y_{R}^{(k)}[t][\lambda]$ denotes the total received optical power at the wavelength $\lambda$ during the time slot $t$ and $R_{P D}[\lambda]$ is the wavelength-dependent responsivity of the PD in $\mathrm{A} \cdot \mathrm{W}^{-1}$. We use $h_{P D}(t)$ to distinguish electrical impulse responses from impulse responses calculated directly by the optical power, i.e. $h(t)$. The channel DC gain determines the achievable signal-to-noise ratio (SNR) for a fixed transmitted power. The RMS delay spread, denoted by $\tau_{R M S}$, is an important measure of inter-symbol interference (ISI), which is a major problem in the design of a robust wireless communication channel. The RMS delay spread $\tau_{R M S}$ of a channel can be calculated using the impulse response [10]:

$\tau_{R M S}=\sqrt{\frac{\int_{-\infty}^{\infty}(t-\mu)^{2} h_{P D}^{2}(t) d t}{\int_{-\infty}^{\infty} h_{P D}^{2}(t) d t}}$,

where $\mu$ is the mean delay spread which is calculated by

$\mu=\frac{\int_{-\infty}^{\infty} t^{2} h_{P D}^{2}(t) d t}{\int_{-\infty}^{\infty} h_{P D}^{2}(t) d t}$.

Similar to the channel DC gain $H 0^{(K)}$, the RMS delay spread with different numbers of total reflections $\tau_{R M S}^{(K)}$ are calculated. Approximate results of $\tau_{R M S}^{(K)}$ can be obtained by replacing integrals with finite summations and substituting $h_{P D}(t)=\sum_{k=0}^{K} h_{P D}^{(k)}[t]$. The values $H 0^{(K)}$ and $\tau_{R M S}^{(K)}$ of all links listed in Table 3 (which are simulated in all environments as listed in Table 1) are calculated.

The accuracy of the DC gain and the RMS delay spread is important for VLC channel characterizations. Thus, it is essential to compare the calculated results of $H 0^{(K)}$ and $\tau_{R M S}^{(K)}$ obtained from different simulation models. To highlight the error caused by increasing $K$, we show differences in the results represented by a calculation error ratio $\xi$. The calculation error ratio of the DC gain is

$\xi_{H 0^{(K)}}=\left|1-\frac{H 0_{\text {const }}^{(K)}}{H 0_{w v}^{(K)}}\right|, \quad H 0_{w v}^{(K)} \neq 0$,

where $H 0_{w v}^{(K)}$ and $H 0_{\text {const }}^{(K)}$ are DC gains calculated from impulse responses simulated in the wavelength-dependent environments and environments with constant reflectivity, respectively. Similarly, $\xi_{\tau_{R M S}^{(K)}}$ can be calculated with (21) substituting $H 0_{w v}^{(K)}$ and $H 0_{c o n s t}^{(K)}$ with $\tau_{w v}^{(K)}$ and $\tau_{c o n s t}^{(K)}$, respectively. Mean values and corresponding confidence intervals (95\%) of $\xi_{H 0^{(K)}}$ and $\xi_{\tau_{R M S}^{(K)}}$ are presented in Fig. 10. It can be clearly seen that the calculation error rates of the DC gain and the RMS delay spread increase with the number of total reflections $K$. The calculation error ratio of the RMS delay spread increases from $3 \%$ to $8.8 \%$ when $K$ increases from 1 to 5 . The same trend can be seen in the calculation error ratio of the DC gain, which increases from $3.4 \%$ to $4.6 \%$ when $K$ increases from 1 to 5 . Although a limited number of links is compared, it is shown by confidence intervals that the use of the model with averaged reflectivity and responsivity increases the calculation error with the total number of 
reflections for most simulation scenarios. Thus, the wavelength dependency of VLC links must be considered in order to obtain accurate results. In addition, it is obvious that the model assuming constant reflectivity and responsivity is inappropriate for simulations taking into account optical devices, for instance, optical filters and amplifiers, which have chromatic properties in the visible light spectrum.

\subsection{Mirror-aided non-LOS VLC channel characterizations}

The signal-to-noise ratio (SNR) is an important measure of the quality of a wireless channel. A higher SNR value implies the ability to achieve higher channel capacity without significant errors. In order to investigate the effect of link configurations on VLC channels, we analyze the SNR of VLC channels modulated with optical orthogonal frequency division multiplexing (O-OFDM), which is a promising modulation scheme for optical wireless channels [27]. Transmitted data is quadrature amplitude modulated to represent the magnitude of the transmitted signal in the frequency domain. The time-domain signal is the inverse discrete Fourier transform of the modulated data symbols and represented by optical power. In DCO-OFDM, DC-bias is added to ensure non-negative optical intensity. The transmitted time-domain signal can be represented by the superposition of time-domain signals transmitted in all subcarriers $X_{n}(t), n=0,1, \ldots, N_{c}-1$, added by the DC-bias $X_{D C}$, where $N_{c}$ is the number of subcarriers. The 0 -th and $N_{c} / 2$ th subcarriers are set to zero, where the $N_{c} / 2$-th subcarrier is the Nyquist term. As the intensity modulated signal must be real, Hermitian symmetry is required [4], i.e. $X_{n}=X_{N_{c}-n}^{*}$, where [.] ${ }^{*}$ is the complex conjugate operation. The DC-bias in DCO-OFDM can be utilized for illumination purposes, which is a suitable candidate for access downlinks and non-LOS backhaul links. In DCO-OFDM, the average transmitted optical power $P_{o p t}$ is the expected value of the transmitted signal $X_{n}(t)$, i.e. the DC bias $X_{D C}$. The total transmitted electrical power is $P_{\text {elec }}=\sum_{n=1}^{N_{c}-1} E\left[X_{n}^{2}(t)\right]$, where $E[\cdot]$ denotes the expectation operator. The relationship between $P_{o p t}$ and $P_{\text {elec }}$ can be obtained by $a=P_{\text {opt }} / \sqrt{P_{\text {elec }}}$ [4], where $a$ is a unit-less bias factor. We assume that the electrical power are equally allocated on $N_{c}-2$ subcarriers, i.e. the electrical power transmitted on $n$-th subcarrier is $P_{n, \text { elec }}=P_{\text {elec }} /\left(N_{c}-2\right), n \neq 0, N_{c} / 2$.

Inter-symbol interference (ISI) caused by large rootmean-square (RMS) delay spread, which denoted by $\tau_{R M S}$, is a major problem in the design of a robust wireless communication channel. In order to avoid the ISI caused by the large RMS delay spread, we add a cyclic prefix (CP) to the transmitted time-domain signals. The length of CP is chosen such that the CP duration is longer than 4 times the RMS delay spread, i.e. $N_{c p}=\left\lceil 4 \tau_{R M S} / T_{S}\right\rceil$, where $\lceil x\rceil$ is the least integer greater than or equal to $x$ and $T_{S}$ is the sampling rate
Table 4: Channel parameters

\begin{tabular}{c|c|c}
\hline Parameter & Symbol & Value \\
\hline Transmitted optical power & $P_{o p t}$ & $10 \mathrm{~W}$ \\
DC bias factor & $a$ & 3 \\
Signal bandwidth & $B$ & $300 \mathrm{MHz}$ \\
Number of subcarriers & $N_{c}$ & 64 \\
Noise spectral density & $N_{0}$ & $1 \times 10^{-21} \mathrm{~A}^{2} \cdot \mathrm{Hz}^{-1}$ \\
\hline
\end{tabular}

which is chosen to be twice the signal bandwidth $B$. The RMS delay spread $\tau_{R M S}$ of a channel can be calculated using the impulse response [10]:

$\tau_{R M S}=\sqrt{\frac{\int_{-\infty}^{\infty}(t-\mu)^{2} h_{P D}^{2}(t) d t}{\int_{-\infty}^{\infty} h_{P D}^{2}(t) d t}}$,

where $\mu$ is the mean delay spread which is calculated by

$\mu=\frac{\int_{-\infty}^{\infty} t^{2} h_{P D}^{2}(t) d t}{\int_{-\infty}^{\infty} h_{P D}^{2}(t) d t}$.

Inserting $\mathrm{CP}$ causes loss in power efficiency and consequently causes SNR loss. We calculate the SNR of each channel taking into account the loss in power efficiency caused by $\tau_{R M S}$ :

$S N R=10 \log _{10}\left(E\left[\frac{P_{n, e l e c}\left|H_{n}\right|^{2}}{\sigma_{n}^{2}}\right]\right)+10 \log _{10}\left(\frac{N_{c}-2}{N_{c}-2+N_{c p}}\right)$,

where $\left|H_{n}\right|$ is the channel magnitude response on the $n$-th subcarrier, $\sigma_{n}^{2}=N_{0} B / N_{c}$ is the noise power on the $n$-th subcarrier, and $N_{0}$ is the power spectral density of the additive white Gaussian noise. The SNR is represented in $\mathrm{dB}$. Parameters of OFDM channels are presented in Table 4.

In order to highlight the effect of transceivers' concentrations and link configurations on the channel performance, we present the SNR of the received signal over each VLC channel normalized to the required SNR to achieve the bit error rate (BER) of $10^{-6}$ when using uncoded fourquadrature amplitude modulation (4-QAM) and 64-QAM. The required $\mathrm{BER}=10^{-6}$ is below the $7 \%$ hard-decision FEC limit of $3.8 \times 10^{-3}$ [28]. The relationship between SNR and BER in additive white Gaussian channel is $B E R \approx$ $\frac{4}{\log _{2} M}\left(1-\frac{1}{\sqrt{M}}\right) \sum_{i=1}^{\sqrt{M} / 2} Q\left(2(i-1) \sqrt{3 \gamma \log _{2} M /(M-1)}\right)$ [29], where $Q(x)$ is the tail probability distribution function, $M$ is the QAM size and $\gamma=E_{b} / N_{0}=\frac{P_{S}}{P_{N}} \frac{R_{b}}{B}$ is the SNR per bit, where $R_{b}$ is the net data rate of OFDM signal exclusive CP and FEC overhead, and $P_{S}$ and $P_{N}$ represent received signal power and noise power, respectively. The ratio between $P_{S}$ and $P_{N}$ is the SNR. The net bit rate can be calculated with $R_{b}=\frac{2 B N_{d} \log _{2} M}{N_{c}}$, where $N_{d}$ is the number of data subcarriers. The net bit rates using uncoded 4-QAM and 64-QAM 


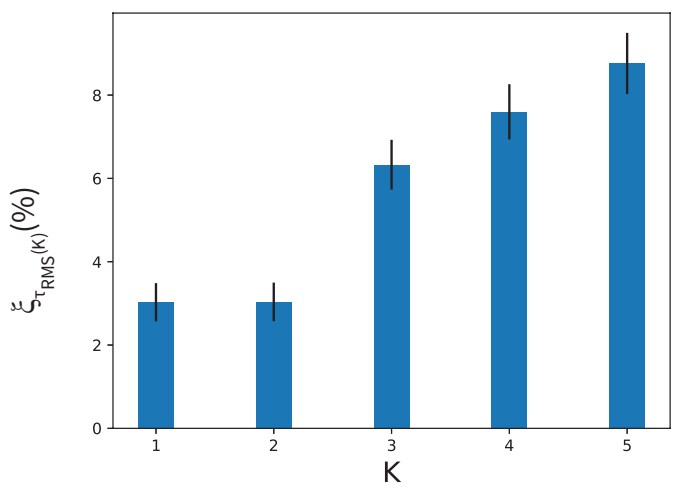

(a)

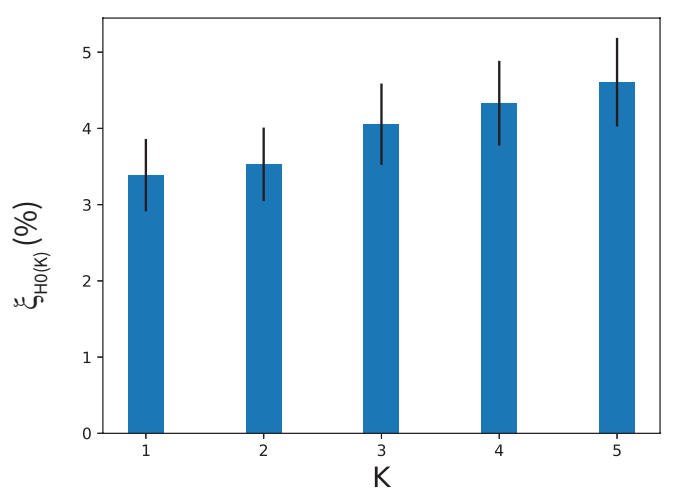

(b)

Fig. 10: Mean values and confidence intervals of (a) the calculation error ratio of the DC gain $\xi_{H 0^{(K)}}$ and (b) the calculation error ratio of the RMS delay spread $\xi_{\tau_{R M S}^{(K)}}$

are 581.25 Mbps and 1.74 Gbps, respectively. The calculation of gross bit rates must take into account the overhead caused by additional CP samples and FEC codes.

SNRs of different link configurations are shown in Fig. 11. It can be seen that increasing $F O V$ can improve the performance of purely diffuse non-LOS channels, while no significant performance enhancement to mirror-aided non-LOS channels when $F O V$ is large enough to receive the first-order specularly reflected signal, which corresponds to $F O V \geq 17^{\circ}$ with Configuration $\mathrm{A}$ and $\mathrm{C}$ and $F O V \geq 0^{\circ}$ with the others. As there are no specular reflections in Environment 1, increasing FOV can slightly strengthen the received optical power. However, in Environment 2 and 3, first-order specular component dominates the channel impulse response. Furthermore, large $F O V$ could lead to performance degradation of some diffuse non-LOS channels. This is likely due to the penalty caused by long CP duration.

It is clearly shown that purely diffuse non-LOS channels with the given channel parameters cannot guarantee reliable transmission with bit rate of 581.25 Mbps, i.e. 4-QAM. Channels in Environment 2 and 3 have larger SNRs than channels in Environment 1, as the received optical power rises when adding first-order specular reflections. Mirroraided non-LOS channels in Environment 2 can support reliable transmission with 4-QAM. However, when the floor is covered by a large mirror, i.e. in Environment 3, concentrate light pattern $\left(m=4.8\right.$, i.e. $\left.\Phi_{1 / 2}=30^{\circ}\right)$ is required to provide sufficient SNR. In addition, mirror-aided non-LOS channels with Configuration A and B cannot guarantee reliable transmission with 64-QAM. By using more directed receivers (Configuration $\mathrm{C}$ and $\mathrm{D}$ ) and transmitters with small semi angle, mirror-aided non-LOS channels in Environment 2 are able to guarantee reliable transmission with 64-QAM. Generally, following requirements are needed to achieve re- liable transmission with large data rate: directed receivers (using Configuration $\mathrm{C}$ and D), small mirror size (Environment 2), and more concentrated senders.

\section{Conclusion}

The aim of this research is to investigate the performance of purely diffuse and mirror-aided non-LOS VLC channels, which are promising candidates for the backhaul transmission of indoor VLC networks. To the best of our knowledge, the proposed non-LOS link-based backhaul solution as well as the effect of link configurations on mirror-aided non-LOS links have not been researched before. Simulations of mirror-aided non-LOS links are undertaken by a time-efficient model based on an iterative method. Multibounce channel impulse responses taking into account both diffuse and specular reflections can be obtained with this iterative model in reasonable time. Simulations of different link configurations are undertaken to assess the performance of non-LOS channels. Simulation results show that adding the first-order specular reflection to non-LOS channels increases the SNR by at least $10 \mathrm{~dB}$, while further increasing the mirror size cannot lead to better channel performance. It is also shown that link configurations have large impact on the channel performance. In our simulation, mirror-aided non-LOS channels with directed receivers and concentrated transmitting light pattern can guarantee reliable data transmission with data rate of $1.74 \mathrm{Gbps}$. Thus, the proposed mirror-aided non-LOS VLC channels have the potential to support high-speed backhaul data transmission. The interference caused by other active VLC APs is not covered in this study, but will be considered in the future work. 
(a) Configuration $\mathrm{A}$

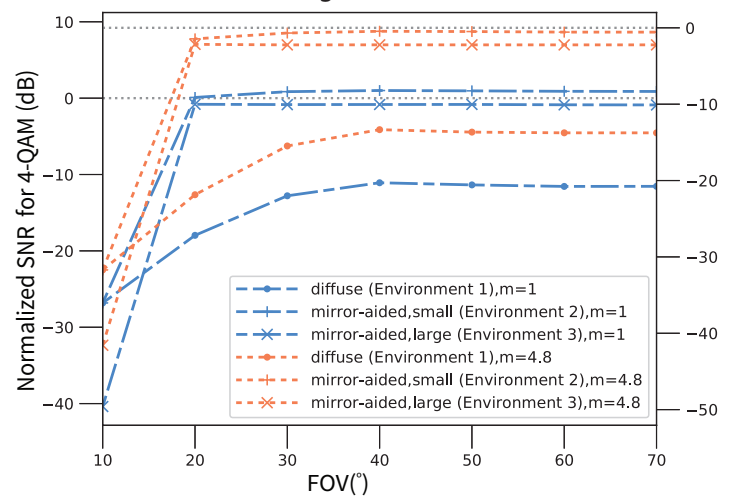

(c) Configuration $\mathrm{C}$

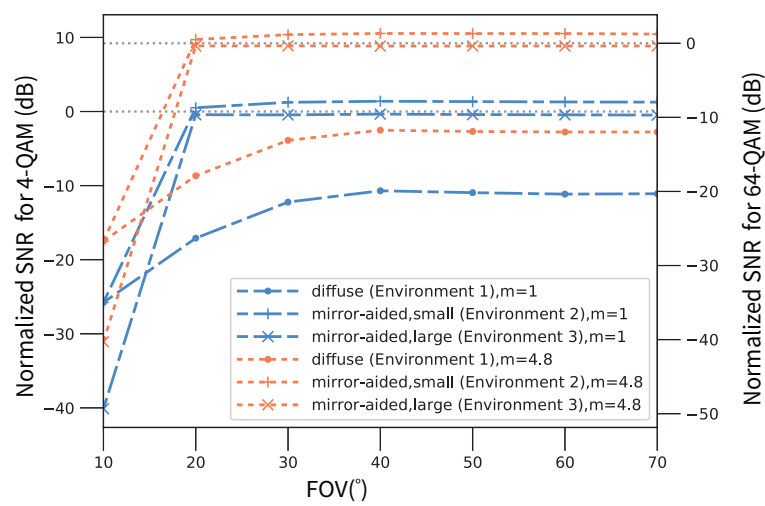

(b) Configuration B

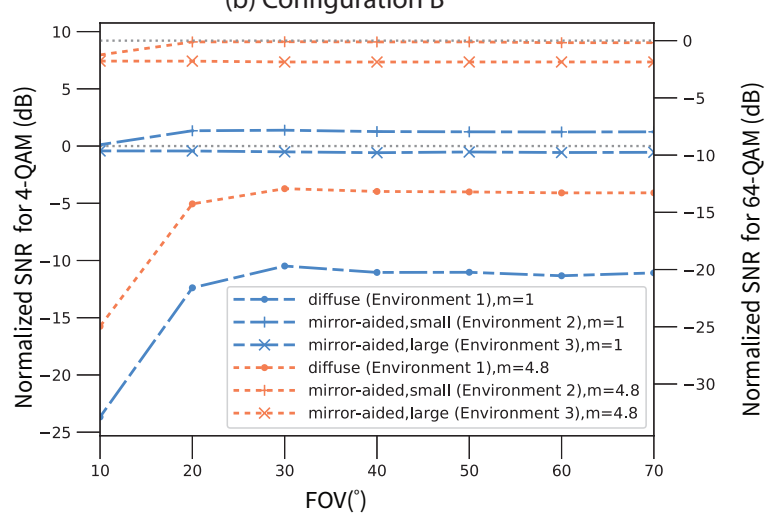

(d) Configuration D

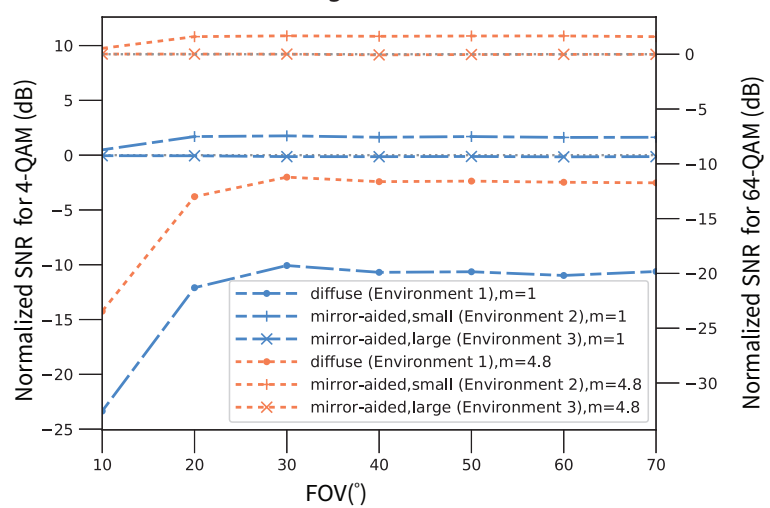

Fig. 11: SNR of non-LOS VLC channels with different link configurations normalized to the required SNR using uncoded 4-QAM and 64-QAM with the BER requirement of $10^{-6}$; (a) $S$ and $R$ oriented directly downwards; (b) $S$ oriented downwards, $R$ oriented towards the mirror; (c) $S$ oriented towards the mirror, $R$ oriented downwards; (d) $S$ and $R$ oriented towards the mirror

\section{References}

1. Y. Tanaka, T. Komine, S. Haruyama, M. Nakagawa. Indoor visible communication utilizing plural white LEDs as lighting, in 12th IEEE Int. Symp. Personal, Indoor and Mobile Radio Communications, vol. 2 (2001), vol. 2, pp. 81-85. DOI 10.1109/PIMRC.2001.965300

2. T. Komine, M. Nakagawa. Fundamental analysis for visiblelight communication system using LED lights. IEEE Transactions on Consumer Electronics 50(1), 100 (2004). DOI 10.1109/TCE.2004.1277847

3. J. Grubor, S. Randel, K.D. Langer, J.W. Walewski. Broadband information broadcasting using LED-based interior lighting. Journal of Lightwave Technology 26(24), 3883 (2008). DOI 10.1109/JLT.2008.928525

4. C. Chen, M. Ijaz, D. Tsonev, H. Haas. Analysis of downlink transmission in DCO-OFDM-based optical attocell networks, in 2014 IEEE Global Communications Conference, GLOBECOM 2014 (2014), pp. 2072-2077. DOI 10.1109/GLOCOM.2014.7037113

5. T. Komine, M. Nakagawa. Integrated System of White LED Visible Light Communication and Power Line Communication. IEEE Transactions on Consumer Electronics 49(1), 71 (2003)

6. P. Hu, P.H. Pathak, A.K. Das, Z. Yang, P. Mohapatra. PLiFi: Hybrid WiFi-VLC networking using power lines, in 3rd ACM Workshop on Visible Light Communication Systems, VLCS 2016, vol. 03-07-Octo (2016), vol. 03-07-Octo, pp. 31-36. DOI $10.1145 / 2981548.2981549$

7. D. Karunatilaka, F. Zafar, V. Kalavally, R. Parthiban. LED Based Indoor Visible Light Communications: State of the Art. IEEE Communications Surveys \& Tutorials 17(3), 1649 (2015). DOI 10.1109/COMST.2015.2417576

8. Y. Wang, N. Chi, Y. Wang, L. Tao, J. Shi. Network Architecture of a High-Speed Visible Light Communication Local Area Network. IEEE Photonics Technology Letters 27(2), 197 (2015). DOI 10.1109/LPT.2014.2364955

9. H. Kazemi, M. Safari, H. Haas. A Wireless Backhaul Solution Using Visible Light Communication for Indoor Li-Fi Attocell Networks, in IEEE ICC 2017 Optical Networks and Systems Symposium (2017)

10. J.B. Carruthers, J. Kahn. Modeling of Nondirected Wireless Infrared Channels. IEEE TRANSACTIONS ON COMMUNICATIONS 45(10), 1260 (1997)

11. C. Chen, D. Basnayaka, H. Haas. Non-line-of-sight channel impulse response characterisation in visible light communications. 2016 IEEE International Conference on Communications, ICC 2016 (2016). DOI 10.1109/ICC.2016.7511382

12. V. Jungnickel, V. Pohl, S. Nönnig, C. von Helmolt. A physical model of the wireless infrared communication channel. IEEE Journal on Selected Areas in Communications 20(3), 631 (2002). DOI $10.1109 / 49.995522$ 
13. F. Lopez-Hernandez, R. Perez-Jimenez, A. Santamaria. Raytracing algorithms for fast calculation of the channel impulse response on diffuse IR wireless indoor channels. Optical Engineering 39, 2775 (2000)

14. E. Sarbazi, M. Uysal, M. Abdallah, K. Qaraqe. Indoor channel modelling and characterization for visible light communications. International Conference on Transparent Optical Networks pp. 14 (2014). DOI 10.1109/ICTON.2014.6876576

15. F. Miramirkhani, M. Uysal. Channel Modeling and Characterization for Visible Light Communications. IEEE Photonics Journal 7(6) (2015). DOI 10.1109/JPHOT.2015.2504238

16. J.R. Barry, J. Kahn, W.J. Krause, E.A. Lee, D.G. Messerschmitt. Simulation of Multipath Impulse Response for Indoor Wireless Optical Channels. IEEE Journal on Selected Areas in Communications 11(3), 367 (1993). DOI 10.1109/49.219552

17. J.B. Carruthers, P. Kannan. Iterative site-based modeling for wireless infrared channels. IEEE Transactions on Antennas and Propagation 50(5), 759 (2002). DOI 10.1109/TAP.2002.1011244

18. K. Lee, H. Park, J.R. Barry. Indoor channel characteristics for visible light communications. IEEE Communications Letters 15(2), 217 (2011). DOI 10.1109/LCOMM.2011.010411.101945

19. F. Lopez-Hernandez, M. Betancor. DUSTIN: Algorithm for calculation of impulse response on IR wireless indoor channels. Electronics Letters 33(21), 1804 (1997)

20. M.A. Khalighi, M. Uysal. Survey on Free Space Optical Communication: A Communication Theory Perspective. IEEE Communications Surveys \& Tutorials 16(4), 2231 (2014). DOI 10.1109/COMST.2014.2329501

21. M. Aubé, J. Roby, M. Kocifaj. Evaluating Potential Spectral Impacts of Various Artificial Lights on Melatonin Suppression, Photosynthesis, and Star Visibility. PLoS ONE 8(7) (2013). DOI 10.1371/journal.pone.0067798

22. A.M. Baldridge, S.J. Hook, C.I. Grove, G. Rivera. The ASTER spectral library version 2.0. Remote Sensing of Environment 113(4), 711 (2009). DOI 10.1016/j.rse.2008.11.007

23. F.R. Gfeller, U. Bapst. Wireless in-House Data Communication Via Diffuse Infrared Radiation. Proceedings of the IEEE 67(11), 1474 (1979). DOI 10.1109/proc.1979.11508

24. C. Schlick. An Inexpensive BRDF Model for Physically-based Rendering (1994). DOI 10.1111/1467-8659.1330233

25. S. Arumugam, J. John. Effect of transmitter positions on received power and bandwidth in diffuse indoor optical wireless systems. Optical and Quantum Electronics 39(1), 1 (2007). DOI 10.1007/s11082-007-9064-x

26. Á. De-La-Llana-Calvo, J. Lázaro-Galilea, A. Gardel-Vicente, D. Rodríguez-Navarro, I. Bravo-Muñoz, G. Tsirigotis, J. IglesiasMiguel. Modeling the Effect of Optical Signal Multipath. Sensors 17(9), 2038 (2017). DOI 10.3390/s 17092038

27. J. Armstrong. OFDM for optical communications. Journal of Lightwave Technology 27(3), 189 (2009). DOI 10.1109/JLT.2008.2010061

28. I.T.S. Group, ITU-T Rec. G.975.1 (02/2004) Forward error correction for high bit-rate DWDM submarine systems. Tech. rep. (2005)

29. J. Lu. M-PSK and M-QAM BER computation using signal-space concepts. IEEE Transactions on Communications (1999). DOI $10.1109 / 26.752121$ 